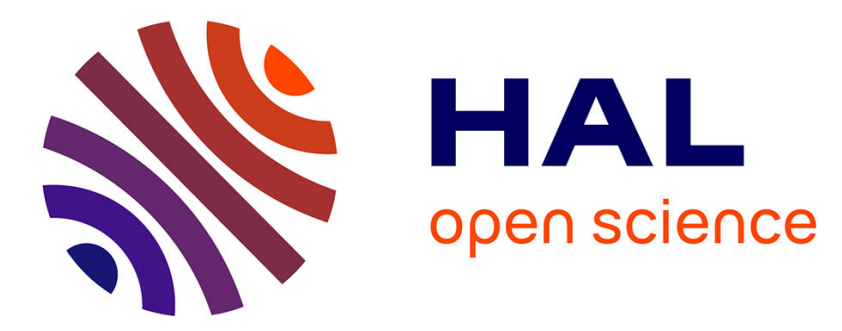

\title{
Allometric shape vector projection: A new method for the identification of allometric shape characters and trajectories applied to the human astragalus (talus)
} W.C.H. Parr, A. Ruto, C. Soligo, H.J. Chatterjee

\section{- To cite this version:}

W.C.H. Parr, A. Ruto, C. Soligo, H.J. Chatterjee. Allometric shape vector projection: A new method for the identification of allometric shape characters and trajectories applied to the human astragalus (talus). Journal of Theoretical Biology, 2011, 272 (1), pp.64. 10.1016/j.jtbi.2010.11.030 . hal00664004

\section{HAL Id: hal-00664004 https://hal.science/hal-00664004}

Submitted on 28 Jan 2012

HAL is a multi-disciplinary open access archive for the deposit and dissemination of scientific research documents, whether they are published or not. The documents may come from teaching and research institutions in France or abroad, or from public or private research centers.
L'archive ouverte pluridisciplinaire HAL, est destinée au dépôt et à la diffusion de documents scientifiques de niveau recherche, publiés ou non, émanant des établissements d'enseignement et de recherche français ou étrangers, des laboratoires publics ou privés. 


\section{Author's Accepted Manuscript}

Allometric shape vector projection: A new method for the identification of allometric shape characters and trajectories applied to the human astragalus (talus)

W.C.H. Parr, A. Ruto, C. Soligo, H.J. Chatterjee

PII: $\quad$ S0022-5193(10)00625-9

DOI: $\quad$ doi:10.1016/j.jtbi.2010.11.030

Reference: $\quad$ YJTBI6252

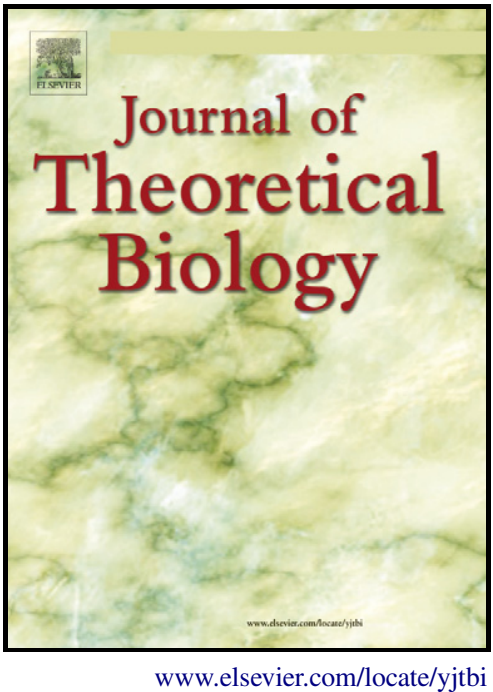

To appear in: $\quad$ Journal of Theoretical Biology

Received date: $\quad 8$ March 2010

Revised date: $\quad 22$ November 2010

Accepted date: $\quad 23$ November 2010

Cite this article as: W.C.H. Parr, A. Ruto, C. Soligo and H.J. Chatterjee, Allometric shape vector projection: A new method for the identification of allometric shape characters and trajectories applied to the human astragalus (talus), Journal of Theoretical Biology, doi:10.1016/j.jtbi.2010.11.030

This is a PDF file of an unedited manuscript that has been accepted for publication. As a service to our customers we are providing this early version of the manuscript. The manuscript will undergo copyediting, typesetting, and review of the resulting galley proof before it is published in its final citable form. Please note that during the production process errors may be discovered which could affect the content, and all legal disclaimers that apply to the journal pertain. 


\section{Title}

Allometric shape vector projection: A new method for the identification of allometric shape characters and trajectories applied to the human astragalus (talus).

\section{Author names and affiliations}

Parr, W.C.H. ${ }^{1,2}$, Ruto, $\mathrm{A}^{3}$, Soligo, $\mathrm{C}^{4}$ and Chatterjee, H.J. ${ }^{1}$

${ }^{1}$ Research Department of Genetics, Evolution and Environment, Division of Biosciences, University College London, Gower Street, London, WC1E 6BT, UK

${ }^{2}$ Department of Palaeontology, Natural History Museum, London, SW7 5BD, UK

${ }^{3}$ Department of Computer Sciences, University College London, Malet Place, London, WC1E 6BT, UK

${ }^{4}$ Department of Anthropology, University College London, 14 Taviton Street, London, WC1H 0BW, UK

\section{Authors email addresses}

Parr: parr.will@googlemail.com

Ruto: tony@ruto.me

Soligo: c.soligo@ucl.ac.uk

Chatterjee: h.chatterjee@ucl.ac.uk

\section{Corresponding Author}

H. J. Chatterjee, Research Department of Genetics, Evolution and Environment, Division of Biosciences, Darwin Building, University College London, Gower Street, London, WC1E 
6BT, UK. Email: h.chatterjee@ucl.ac.uk; Tel: +44(0)20 76794113; Fax: +44(0)20 76797096.

\begin{abstract}
The surface morphology of the human astragalus (talus) is difficult to represent accurately using landmarks as it is essentially globular in shape. Advances in laser scanning technology allow fast and accurate capture of bone surface morphology. However, methodologies to utilise these new accurate 3D data have not been fully developed. The present study uses canonical sampling of whole surface morphology attained through laser scanning and for the first time applies the technique to analysis of bone morphology. We introduce a new technique for identifying allometric shape characters in whole bone surface morphology. In a sample of adult human astragalus the new technique is successful in identifying and isolating intra-specific allometric shape characters in a bone which typically lacks landmarks and has, consequently, proved difficult to analyse using traditional 3D morphometric methods.
\end{abstract}

\title{
Keywords
}

scaling; canonical sampling; laser scanning; principal components analysis; allometry 


\section{Introduction}

The geometric morphometric method combines Galton's multivariate techniques, which are based on covariance matrices of a number of measurements designed to capture the metrics of a biological object, with D'Arcy Thompson's direct visualisations of simple geometric transformations leading to differing biological forms [1]. The key to the geometric morphometric method is to identify discrete homologous 'landmark' points, which can be identified reliably across the sample. Landmarks enable individual biological forms to be described as well as mathematically defining the morphological transformations that warp one form to the shape of another. However, in complex biological forms such as post-cranial bones and whole bodies, and particularly in three dimensional analyses, truly homologous landmarks are difficult to locate in sufficient abundance to accurately describe the form of the object [2]. In such cases Bookstein [3] advocates the use of type III landmarks. Gunz et al [4] and Mittereocker et al [5] have applied type III semi-landmarks across the surface of skulls to create slid semi-landmarks $[5]$.

The use of the landmark based methods of geometric morphometrics for describing shape differences between specimen samples habitually contains the following steps $[6,7,8$ $9,10]$ : selection of homologous landmark points on all specimens within a sample, alignment of specimens by standardisation (centering by translation, rotational transformation and scaling) of landmark coordinate points before examination of the remaining shape differences between specimens by Principal Components (PC) analysis [9]. For 2D and 3D landmarks and semi-landmarks, scaling is commonly achieved by 
division of coordinates by the centroid size, as described in Gower [10] and Rohlf and Slice [9]. The centroid of each specimen is calculated as the mean of the specimen's coordinates and the centroid size is represented by the squared root of sum of squared distances to each of the coordinate points from the centroid [10]. Scaling aims to remove the effects of geometric scaling in the data. Any residual differences in the measurements or coordinate data of different specimens after translation, scaling and rotation will therefore correspond to shape rather than shape, size and orientation [10] as shape is defined as the aspects of form remaining once size has been removed $[11,12]$. However, in some cases, such as analyses concerned with intra-specific allometric shape characters, it may be advantageous not to scale the data sets before performing PC analysis. If an analysis of the un-scaled data is performed, then PC 1 is likely to identify 'size' as the major difference between bone specimens in a sample. Crucially however 'size' will incorporate geometric size differences between specimens and also all of the size correlated morphological differences in the specimens $[13,14,15]$.

Harcourt-Smith [6] and Hellier and Jeffrey [7] analysed allometry in the astragalus of human and non-human apes. Both studies took landmark and semi-landmark 3D coordinates as their initial data. Following Procrustes superimposition (translation, rotation and scaling), a PC analysis was performed and the size proxy (centroid size in HarcourtSmith [6] and femur length in Hellier and Jeffrey [7]) was correlated with PC scores. In this way, the authors determined how much of the shape difference captured in each PC was the result of size differences between astragali, i.e. reflected allometric shape variation. This methodology generally resulted in relatively low correlations $(r<+/-0.4)$ between size and individual PCs and many PCs being minimally correlated with size. Therefore, no single 
PC identified shape differences in the astragali that were solely the result of size differences. This makes it difficult to identify exactly which shape differences in the astragali are the results of size differences alone.

\subsection{Aims}

This paper aims to further the geometric morphometric tool set available to biologists interested in understanding 3D allometric shape change. As Van der Linde and Houle [12] point out, biologists frequently use visualisations of the patterns of covariances identified by their morphometric analyses to understand the nature of the variation in biological shapes they are examining. The method introduced here has been specifically designed to allow visualization of complex 3D shape changes that are related to differences in size (i.e. allometric shape changes) in bones, or other 3D objects, that do not have many readily identifiable Type I or II landmarks [3] that are truly biologically homologous and can be repeatedly located across most of their surfaces throughout a sample [2]. The subject of this study is the modern human astragalus; a particularly globular bone, which is devoid of Type I landmarks $[6,7,16]$. The new methodology presented in this study of canonical sampling of whole surface morphology in conjunction with allometric vector identification and projection aims to isolate allometric shape characters in adult human astragali.

\section{Methods}

The astragali examined for this study were housed in the Department of Palaeontology at the Natural History Museum (NHM) in London. A total of 59 adult 
human astragali were included in the study, with 24 from confirmed males, 29 from confirmed females and 6 unknowns. Male and female status was confirmed either by grave records or by gender records determined from skeletal morphology and kept by the NHM. Specimens were chosen that did not show any osteological signs of pathologies such as bone breakages or osteoarthritis. Table 1 shows the population breakdown of the astragali used in this study. The sample was composed of different ethnic groups as the aim of this study was to identify general allometric shape characters that apply to the species Homo sapiens, rather than of a specific ethnic group. Adult specimens were selected as having full epiphyseal fusion of the long bones.

A Konica Minolta Vivid 910 surface laser scanner was used to capture the entire surface morphology of each astragalus. The scanner is accurate to $\mathrm{X}: \pm 0.22 \mathrm{~mm}, \mathrm{Y}$ : $\pm 0.16 \mathrm{~mm}, \mathrm{Z}: \pm 0.10 \mathrm{~mm}$. Surface data are initially captured and processed using the scanner's associated software package, Polygon Editing Tool [17] before being further edited in RapidForm 2006 [18]. The initial astragalus scans resulting from PET would often have small holes or other scanning artefacts due to the merging process not succeeding in integrating the initial scans flawlessly. These holes were filled using RapidForm 2006 [18] with either the hole filling tool or the defeature tool (for scanning artefacts). The hole filling and defeature tools use the curvature of the model around the hole or scanning artefact to re-mesh the model's surface so that the result is consistent with the surrounding model's surface. The resulting shells (Figure 1), or virtual 3D bones, are made up of point clouds and triangles. The points have $\mathrm{x}, \mathrm{y}, \mathrm{z}$ coordinates in $3 \mathrm{D}$ space, and the triangles are created by joining these 3D points (for detailed method see Parr [15]). 


\subsection{Canonical sampling}

The canonical sampling process aims to give an accurate representation of the whole surface morphology of astragali. The procedure for canonically sampling the astragali is as follows. The bones are aligned with their length running along the $\mathrm{x}$-axis (in 3D space), their widths running along the y-axis, and their depths along the $\mathrm{z}$-axis. This is achieved by rotating each model so that the principal axes are aligned with the global 3D axes, with principal axis 1 aligned to the global $\mathrm{x}$-axis, principal axis 2 aligned with the global y-axis, and principal axis 3 aligned with the global z-axis. All remaining differences in orientation of the bones are then minimised by ICP (Iterative Closest Point) registration of all of the specimens [19]. This process is similar to a Procrustes registration, except that the ICP algorithm does not need each bone model to have the same number of points, or the points to be homologous [19]. ICP registration relies on matching the surface morphology of the bone models and ensures that they are in similar orientations. Note that at this point the astragali models have not been scaled. Each 3D bone model is then divided up into 50 slices along its length. Each of these 50 slices is then sampled 50 times along its circumference; this forms the canonical sample [14, 20, 21]. Bone models of varying length are still sampled into the same number of slices (50), with the slices spread evenly from the posterior to the anterior extremes of the bone model [13, 14, 20,21]. Slices of varying circumference are sampled into the same number of radial points (50) based on a fixed radial segmentation. Therefore, repeating the sampling process always produces the identical result as each bone is sampled using the identical slice and radial segmentation process [13, 14]. This canonical sampling process was described by Ruto [14], Ruto et al. 
[13], Buxton et al [20], Douros et al [21]. The process is different from processes used by Polly [22], Gunz et al [4], Mittereocker et al [5], which are variations of template optimisation, in that rather than laying a grid over one aspect of a bone's surface, the canonical sampling process uses the 3D points that make up the original bone model's surface. The canonical representations for this study are made up of 50 rows of canonical points, with each row having 50 points in it, resulting in an initial 2500 canonical points describing the whole bone surface. The canonical samples were sub-sampled (every third canonical point dropped) to reduce point density, but not to the extent of loosing overall surface morphology resolution. To avoid over-sampling the posterior end, which is narrower than the rest of the astragalus, the first row was further point reduced by keeping only every fourth point. The final canonical samples used consisted of 1617 points [15].

The canonical points are spatially rather than biologically homologous. For example, canonical point 1 in the 25 th row of the canonical sampling cylinder will always be half-way along the length of the 3D virtual bone model. This is different to landmarks or pseudo-landmarks for which the stated aim is to be biologically equivalent, but is similar to slid semi-landmarks [2]. However, the spatial equivalence of the canonical points conveys biological meaning in a similar way as traditional linear measurements of distances, where these are not anchored at either end by structurally homologous points (such as, e.g., maximal cranial width). For instance, it is conceivable that someone could describe a particular projection on a bone in a ratio form: in specimen A the projection occurs at $1 / 4$ the length of the bone, whereas in B the same projection occurs at 3/4 the length of the bone. Similarly if there is a difference in the position of a canonical landmark between specimens A and B, we know this corresponds to a difference in morphology between 
bones $\mathrm{A}$ and $\mathrm{B}$, which allows for quantification of differences in the surface morphologies of $\mathrm{A}$ and $\mathrm{B}$.

\subsection{Acquiring size related shape (allometric) characteristics}

The resulting canonical models were Procrustes aligned (translated and rotated, but not scaled). PC analysis was performed on the un-scaled canonical models. The expectation was that the first PC identified by this analysis would represent size related shape variation, including both geometrically and allometrically scaled characters. The extent to which PC 1 identifies size related shape variation was tested by regression analysis of specimen PC 1 scores on centroid size, with a high $\mathrm{R}^{2}$ value ( $\left.>0.9\right)$ indicating a strong correlation with size. In order to test for a major influence (other than size) on residual variation in PC1 scores, we performed t-tests on the means of the residual values for males vs. females and for individuals from populations that are typically shod vs. those from populations that are typically unshod. Next, the eigenvectors for PC 1 were used, in conjunction with the maximum and minimum PC 1 scores, to calculate and draw the PC 1 positive and negative extreme astragalus shapes. This concluded the first stage of the analysis, resulting in a large bone shape and a small bone shape.

The second stage of the analysis was to identify the allometric shape characters.

This was achieved by first performing a Procrustes size adjustment followed by rotational transformation and translation of the two (i.e. large and small) PC 1 extreme shape models defined in the first stage $[9,10]$. Following this step the two PC 1 extreme shape models have a centroid size of 1 , have the same orientation, are centred on the origin $(\{x, y, z\}=$ $\{0,0,0\})$ and consist of 1617 spatially homologous 3D coordinate points. All calculations 
were performed in Mathematica [23] using code developed according to algorithms in Rohlf and Slice [9]. At this stage the size adjusted PC 1 extreme shape models were in 3D point cloud form. For visualisation purposes these can be converted to 'solid' polygon models by triangulation between the 3D coordinate points. This was performed in Rapidform 2006 [18].

The third stage of the analysis assessed which areas of the size adjusted PC 1 extreme shape models exhibit the greatest degrees of size correlated shape change. This was achieved by first calculating the magnitudes of all of the distances between the homologous points. Following this, the 250 homologous points which showed the greatest differences in 3D position, as indicated by having the greatest magnitude of distance, were selected. 3D arrows were then drawn between all of these 250 homologous points, starting at what was the original 'small' PC 1 extreme shape and ending at what was the original 'big' PC 1 extreme model. Only the largest 250 vectors were selected for illustration, as displaying all 1617 vectors would have been visually confusing. This effectively created a vector map of the areas of the PC 1 astragali models exhibiting the most pronounced size related (allometric) shape changes, and indicated in which 3D direction these allometric shape changes occur. Examination of the allometric vector maps, rather than the movements of individual landmarks, conveys the information about the allometric shape characters. This is because the canonical points and allometric vector maps give information on spatial differences between the surfaces of astragali models rather than movements of biologically homologous points on the bones differences as is the case with true landmarks. 


\subsection{Allometric vector identification and extrapolation method validation}

To test whether using the allometric projection as the model for the shape of an astragalus of a particular size is more accurate than using the mean astragalus shape of a sampled population, additional astragali models were created. These models were created by warping the mean astragalar shape for the canonically sampled astragali along the allometric vectors to create an astragalus shape predicted by the allometric vectors for each original canonical astragalus according to its size. We can then ascertain to what degree (mathematically) the shape of an adult human astragalus can be predicted using the allometric vector projection technique. The model pairs (original canonical astragalus and allometric projection) were Procrustes aligned with one another (translated and rotationally transformed but not scaled as the model pairs have the same centroid size) before the mean squared difference (MSdiff) in canonical landmark positions were calculated. The MSdiff is an overall (average) measurement (in $\mathrm{mm}$ ) of how close two models are to one another in shape. In the present analyses, MSdiffs vary from 0.67 to $2.50 \mathrm{~mm}$. The MSdiff was also calculated for the original models paired with scaled mean shape models (these model pairs were Procrustes rotated and translated and had the same centroid sizes). MSdiffs varied from 0.84 to $3.07 \mathrm{~mm}$; greater maxima and minima than for the allometric projections paired with the original models. In 47 out of the 59 models (80\%) the MSdiffs were lower between the Procrustes aligned original and their corresponding allometric projection than between the same Procrustes aligned original and the mean shape, showing that in the majority of cases the astragalus model created by allometric projection was closer than the mean astragalus model's shape to the actual astragalus model's shape. 
To test for differences in allometric vectors between males and females, which could, for example, result from the subtle differences in gait between males and females [24], the male and female astragali were analysed separately. The PC scores were regressed against centroid size separately for the male and female groups. This suggests that projection along allometric vectors can be used to create more accurate models than a species mean shape in cases where an allometric stage is not documented and, hence, both justifies the use of allometric shape vector projections as a tool for quantifying allometric shape change in relatively feature-less bones such as the astragalus and opens the possibility of extrapolating allometric shape variation beyond the range of existing samples.

\section{Results}

\subsection{Method validation}

Principal Components Analysis of un-scaled (but translated and rotated) canonically sampled astragali resulted in the first 10 PCs describing $90.75 \%$ of total variance. Of these, PC1 described $71.18 \%$ of total variance, followed by PC2 with $8.67 \%$ and PC3 with $2.18 \%$ of total variance. Regression of PC 1 against centroid size resulted in a very strong correlation $\left(\mathrm{R}^{2}=0.999\right.$, confidence level $\left.=0.99\right)$ suggesting that PC 1 is strongly associated with size and size correlated shape variation (Figure 2). Regressions of PCs 2-10 gave very low $\mathrm{R}^{2}$ values $\left(\max =\mathrm{PC} 2: 0.00221, \min =\mathrm{PC} 7: 2.12 \times 10^{-7}\right)$. This suggests that PC 1 captures nearly all of the total size related shape variation. For the separate analyses of male and female astragali, Figure 3 shows that in both cases PC 1 identifies size and size correlated shape (male $\mathrm{R}^{2}=0.997$, female $\mathrm{R}^{2}=0.998$ ) and that males are larger than females as the $\mathrm{y}$ intercept of the regression line for males is -12.594 [99\% CIs $=-13.013$, - 
12.175] as opposed to females where the y intercept $=-10.914[99 \%$ CIs $=-11.158$, 10.670]). Additionally, the scaling exponents for the regression lines (male $=0.0148[99 \%$ CIs $=0.0143,0.0153]$, female $=0.0147[99 \%$ CIs $=0.0144,0.0151])$ are very similar $($ not significantly different at a $\mathrm{P}=0.01$ level). This indicates that the relationship of PC 1 with centroid size is essentially the same in both male and female groups.

\subsection{Results of the method as applied to adult human astragali}

The variation in size between the two extreme shapes shown in Figure 4 indicates that PC 1 isolated size differences in the astragali. The allometric shape characters identified by PC 1 for the astragalus are illustrated by the diagrams shown in Figures 5, 6 and 7. In Figures 5 and 6 the 250 canonical landmarks that differ the most between the PC 1 extreme shape models are identified by red arrows running from the blue (original small model) to the yellow (original big) model. These groupings of arrows (the allometric vector maps) illustrate the main regions of allometric shape differences between small and large astragali models.

The main regions of allometric shape differences, from the dorsal view, are: the lateral head, medial facet and medial trochlea, the anterior lateral trochlea, the lateral projection and posterior trochlea regions of the astragalus (see the clustering of red 3D arrows in the dorsal view of the size adjusted PC 1 extreme shape models as shown in Figure 5A and see Figure 1 for annotations for the different facets and regions of the astragalus; see Video 1 for an animation of the allometric shape change from the dorsal view). From the plantar view the allometric shape variations are: the posterior and anterior 
calcaneal facet regions, the posterior region of the sustentaculum facet, the medial region of the head and the lateral projection regions of the astragalus (see Figure 5B and Video 2). From the anterior view (Figure 6A) the main regions of allometric shape difference are the lateral head region, the medial facet and the anterior lateral region of the trochlea facet. From the posterior view (Figure 6B and Video 3) they are the anterior and posterior regions of the calcaneal facet and the posterior regions of the trochlea and lateral facets. The main regions of allometric shape differences from the medial view (Figure 7A), are: the posterior region of the trochlea facet and the anterior region of the medial facet and from the lateral view (Figure 7B and Video 4): the posterior calcaneal facet and lateral head (and neck) regions.

\section{Discussion}

\subsection{Canonical sampling}

The methodology featured in Adams et al [5] and Gunz et al [2], described in Mitteroecker and Gunz [4], used, a variation of template optimisation, which consists of decimating the 3D point cloud of a reference skull until it is represented by only a few roughly evenly spaced semi-landmarks and warping this template to the shape of another specimen. An important difference between template optimisation and canonical sampling techniques is that canonical sampling gives a more accurate representation of an object because the final subset of data is a subset of each object's original points. Template optimisation gives as close an approximation to this as possible, based on optimising the shape of a template to match the object. This optimisation process finds a least squares fit for corresponding landmarks on template and object, while maintaining the template's 
topology (connectivity or relationship between the template's points). This connectivity can limit the positioning accuracy of a template's landmarks on an object and thus lead to a less accurate object representation when compared against the object's original point set

For the purposes of object shape representation, each method is slightly different as outlined below.

Template Optimisation (TO):

1. TO provides a means of placing landmarks on a shape based on a predefined template with an existing topology.

2. The resulting landmarks are approximate positions on the target shape (in other words, the landmarks are not taken from the original model) but are optimised to give a least squares fit between the template shape and target object shape.

3. When assessing the shape variation of a large sample, the general shape variability of the sample can be captured using template representations of the data. However, this variability will include the representation error of the template optimisation technique used [14].

Canonical sampling (CS):

1. CS provides a sample of an object's original data at predefined locations. Where no data are present at those locations, an approximation is used. It is important to note that highly accurate initial models, such as those achieved by the surface laser scanner used in this study, result in more accurate canonical representations essentially containing a larger proportion of the original data points.

2. Canonical sampling does not produce a representation error other than when approximate points are used. 
3. When used for assessing shape variations, canonical sampling relies on the object shapes being similar so that sampling them at specific locations will result in representations that are similar in terms of the regions of whole shape they represent. The PC analysis will then capture the shape variability in these regions. This is the essence of the spatial homology of the canonical landmarks and why allometric vector maps are important rather than movements of individual landmarks. Template optimisation is reliant on choosing a suitable density of landmarks for each region of the original template specimen.

4. Variability between models results from actual shape variation between specimens and the representation error of the sampling procedure. The models generated by canonical sampling have a smaller representation error and hence provide a more accurate shape representation [14]. Ruto [14] found that for human whole body shape in a sample that would be expected to have the same shape variability, the template representation model has a larger total variation (or entropy) than the canonical representation model, thus indicating the representation error was larger in the template representation model. This confirmed that canonical sampling was a more accurate technique for assessing body shape variation. Hence, for sampling of bones such as the astragalus or 3D objects such as whole bodies [14] which are naturally devoid of both Type I and Type II landmarks [3] canonical sampling can be preferable to template optimisation.

\subsection{Identification of allometric vectors and their use to create allometric projections}

With the allometric vector method presented here, it is the allometric vector maps, which are created by drawing the 250 allometric vectors with the greatest magnitude, rather 
than individual landmarks, that elucidate size correlated shape changes which occur in the sample. The allometric vector maps are virtually identical if the scaled PC 1 extreme shape models are not re-aligned (results not presented). However, we recommend performing the second alignment step so as to minimise any orientation differences in the PC 1 extreme shape models. This re-alignment may not be necessary, if there are no size correlated orientation differences in the canonical representations, but it effectively minimises the magnitudes of the allometric vectors. We advise against the use of sub-set superimposition in the case of allometric vector identification [12]. This is because sub-set superimposition, as with Procrustes resistance fit superimposition, is designed to align specimens using the landmarks that are not influenced by allometry $[9,12,25,26]$. However, as we identify allometric shape variation for all of the canonical landmarks, none of them can be considered suitable for use in subset superimposition. Additionally, affine superimposition is not suitable in the case of the allometric shape characters presented in this paper, as there is no obvious allometric affine transformation occurring in any axis of the astragalar shape $[9,12]$.

How well the allometric projections approximated the shape of the original canonical models was calculated as the mean squared difference (MSdiff) between the location of the canonical landmarks in the original and allometric projection model. In the 12 cases where the mean shape had a lower MSdiff than the allometric projection, the difference in MSdiff between the mean and allometric projection was very slight (often 1 or 2 orders of magnitude lower than the average difference in MSdiff between the mean and allometric projection models). This indicates that using allometric projection as the model for the shape of an astragalus of a particular size is usually more accurate than using the 
mean astragalus shape of the sampled population. Frequently, in cases where the MSdiff for the allometric projection was greater than for the mean shape, the original astragalus exhibited fairly unique shape characters not habitually observed in the remainder of the sample.

\subsection{Application of allometric vector and projection techniques in adult human astragali.}

\subsubsection{Intra-specific allometric shape characters identified in the human astragalus.}

Figure 5A (dorsal view) shows that the anterior medial region of the trochlea facet and anterior lateral region of the astragalar head exhibit allometric shape change. These changes lead to the apparent decrease in horizontal astragalar neck angle with increasing size, as described by Day and Wood [27] and Lewis [16] and reduced medial facet curvature observed in the larger (yellow) model in Figure 4 when compared to the smaller (blue) PC 1 extreme shape model. The biomechanical consequence is that the path of force transmission is aligned across the trochlea facet, through the astragalar neck, to the head facet and on to the navicular, as described by Day and Wood [27]. Further, the reduced medial facet curvature causes less medial movement of the tibia during dorsi-flexion of the foot (during the pre toe-off stance phase of the gait). These both potentially lead to comparatively less strain in the astragalar neck of the larger individuals (as suggested by Gebo and Schwartz [28]). The plantar view shows that the anterior (eversion) half of the calcaneal facet appears relatively smaller and re-orientated in the larger (yellow) model whereas the posterior (inversion) half of the facet appears expanded and re-orientated in the big (yellow) model (inversion and eversion facet halves defined as in Wang et al [29]). 
From this analysis alone, it is difficult to conclude what the cause of this allometric shape change is and if they have any functional consequences.

\subsubsection{Male versus female allometric vector maps}

The medial expansion of the anterior medial region of the trochlea and the consequent medial displacement of the medial facet identified in the analysis of the combined male and female samples is not identified as one of the major allometric shape differences in the dorsal view of the male allometric vector map (Figure 8A). Instead, the lateral shift (with increased size) of the medial region of the astragalus head is identified as the region of the medial aspect of the astragalus with the strongest allometric shape difference. The male allometric vector map shows that with increased size the astragalus head becomes re-aligned with the trochlea body and trochlea groove by moving laterally, rather than the trochlea body expanding medially. The lateral expansion of the head in the larger male specimens is possibly a late onset allometric growth pattern as it does not seem to 'outweigh' the medial expansion of the anterior trochlea region in the whole sample analysis. If the astragalus head and trochlea alignment is a functional requirement for efficient and safe (with respect to strains generated in the astragalus) bipedal locomotion in large individuals, then this realignment may be more extreme in larger males compared to both smaller males and females $[27,28]$. This is because males will experience greater force transmission through the tarsal region due to greater body mass compared to females.

The plantar views in Figure 5B and 6 show that the lateral region of the calcaneal facet does not exhibit as much allometric shape change in the female astragali as it does in the male astragali and in the whole human sample. As a result, the calcaneal facet appears 
relatively larger with respect to overall astragalus size in the females and smaller males than in the larger males (Figure 8). Similarly, the posterior region of the sustentaculum facet shows more allometric change in the whole sample and the male specimens than the female sample. These differences in allometric trajectories may be the result of ossification and growth cessation of the astragalus occurring considerably earlier than growth cessation of other foot bones and overall body growth cessation $[30,31]$ and in different processes controlling the overall size of the astragalus (proposed to be genetic control) compared to the size of articular facets (epigenetic; the need to fit with the morphology of their articular partner). This is true in males compared to females [30, 32], and potentially larger males compared to smaller males $[30,32]$. In such a scenario, larger males would grow for even longer than the smaller males (growth duration hypermorphosis [32]) after astragalar growth cessation (when the morphology of the astragalar calcaneal facet is fixed). This results in the differences in allometric characteristics observed in the male compared to female calcaneal facet. Thus, Figure 8 shows that although the size correlated shape and allometric vectors identified in males and females are similar, there are differences.

\section{Conclusion}

The method developed in this study, of identifying size related, allometric, shape characteristics in canonically sampled whole bone models, was successful in identifying and isolating intra-specific allometric shape characters in the adult human astragalus, a bone which typically lacks type 1 landmarks and has, consequently, proved difficult to analyse using traditional 3D morphometric methods. The method first Procrustes rotates and translates (but does not scale at this stage) the canonical representations. PC analysis is 
then performed on the un-scaled, but otherwise aligned, models. PC 1 extreme shapes are calculated and then Procrustes superimposed (rotated, translated and scaled). The 250 largest allometric vectors between spatially homologous canonical points in the scaled (originally) small and (originally) large models are then calculated and displayed on the Procrustes superimposed PC extreme shapes, creating allometric vector maps. These vector maps, rather than individual landmarks, allow for the identification of the main regions, directions and amplitudes of allometric shape change in relatively featureless bones such as the astragalus. Our new method differs from previous methods, which Procrustes scaled (and aligned) data before performing Principal Components (PC) analysis. In such analyses, the size related shape characteristics are frequently spread through a number of PCs, all of which typically show fairly low correlations $(\mathrm{r}<+/-0.4)$ with the size (or body mass) proxy, making it hard to determine exactly which shape characteristics are purely size related.

\section{Authors contributions}

WCHP conceived of the study, undertook all data collection and analyses, and prepared the manuscript. AR performed the canonical sampling of the initial 3D scans and advised with respect to the use of principal component analysis. HJC and CS participated in data interpretation and manuscript preparation and oversaw supervision of the research. All authors have approved the final article. 


\section{Acknowledgements}

The authors gratefully acknowledge NERC (Award number: NER/S/A/2004/12187) and the Natural History Museum, London, for additional funding through a CASE award. The authors are extremely grateful to all of the Palaeontology curators at the Natural History Museum who generously provided access to material used in this study and allowed the use of their departmental Konica Minolta vivid 910 laser scanner. In particular, we are grateful to Robert Kruszynski for extra assistance with the specimens. Further, thanks to Andrew and Helen Parr for recent support enabling this work.

\section{References}

[1] Thompson, D. W., 1917. On Growth and Form. Cambridge University Press.

[2] Gunz, P., Mitteroecker, P., Bookstein, F., 2005. Landmarks in Three Dimensions. In Slice, E. (Ed) Modern Morphometrics in Physical Anthropology, Kluwer Academic/Plenum Publishers, New York.

[3] Bookstein, F. L., 1991. Morphometric Tools for Landmark Data: Geometry and Biology, Cambridge University Press, Cambridge (UK), New York.

[4] Mitteroecker, P., Gunz, P., 2009. Advances in Geometric Morphometrics. Evolutionary Biology, 36, 235-247.

[5] Adams, D. C., Rohlf, F. J., Slice, D. E., 2004. Geometric Morphometrics: Ten Years of Progress Following the 'Revolution'. Italian Journal of Zoology, 71, 5-16.

[6] Harcourt-Smith, W. E., 2002. Form and Function in the Hominoid Tarsal Skeleton. Anthropology. London, UCL.

[7] Hellier, C. A., Jeffery, N., 2006. Morphological Plasticity in the Juvenile Talus. Foot and Ankle surgery, 12, 139-147. 
[8] O'Higgins, P., 2000. The study of morphological variation in the hominid fossil record: biology, landmarks and geometry. Journal of Anatomy, 197, 103-120.

[9] Rohlf, F. J., Slice, D., 1990. Extensions of the Procrustes method for the optimal superimposition of landmarks. Systematic Zoology, 39, 40-59.

[10] Gower, J. C., 1975. Generalized Procrustes analysis. Psychometrika, 40, 33-51.

[11] Mosimann, J. E., 1970. Size allometry: Size and shape variables with characterizations of lognormal and generalized gamma distributions. Journal of the American Statistical Association, 65 (330), 930-945.

[12] Van der Linde, K., Houle, D., 2009. Inferring the Nature of Allometry from Geometric Data. Evolutionary Biology, 36, 311-322.

[13] Ruto, A., Lee, M., Buxton, B., 2006. Comparing Principal and Independent Modes of Variation in 3D Human Torso Shape using PCA and ICA. ICA research network international workshop, University of Liverpool.

[14] Ruto, A., 2009. Dynamic human body modeling and animation. UCL, University of London.

[15] Parr, W. C. H., 2009. Evolutionary and functional anatomy of the hominoid astragalus-new approaches using laser scanning technologies and 3D analyses. UCL, University of London.

[16] Lewis, O. J., 1989. Functional Morphology of the Evolving Hand and Foot, Oxford, Clarendon Press.

[17] PET, 2006. Polygon Editing Tool. 2.20 ed., Konica Minolta.

[18] INUS Technology, 2006. RapidForm 2006, Inus Technology.

[19] Besl, P. J., Mckay, N. D., 1992. A method for registration of 3-D shapes. Ieee Transactions on Pattern Analysis and Machine Intelligence, 14, 239-256.

[20] Buxton, B., Tahan, A., Ruiz, M. C., 2003. Point distribution models of human body shape from a canonical representation of 3D scan data, In 3D Modelling 2003, Paris France, April 2003. 
[21] Douros, I., Ruiz, M. C., Buxton, B. F., Treleaven, P. C., 2002. Web-based software tools for 3d body database access and shape analysis. In numerisation 3D - Scanning, Paris, France, April 2002.

[22] Polly, P. D., 2006. Adaptive zones and the Pinniped ankle. A three dimensional Quantitative analysis of Carnivoran Tarsal Evolution. In Sargis, E. J., Dagosto, M., (Eds.) Mammalian Evolutionary Morphology: A tribute to Frederick S. Szalay. New York, Kluwer/Plenum.

[23] Wolfram Research Inc., 2007. Mathematica, Version 6.0, Champaign, IL.

[24] Wang, W., Crompton, R. H., Carey, T. S., Gunter, M. M., Li, Y., Savage, R., Sellers, W. I., 2004. Comparison of inverse-dynamics musculo-skeletal models of AL 288-1 Australopithecus afarensis and KNM-WT 15000 Homo ergaster to modern humans, with implications for the evolution of bipedalism. Journal of Human Evolution, 47, 453-478.

[25] Rohlf, F. J., 1999. Shape statistics: Procrustes Superimpositions and Tangent Space. Journal of Classification, 16, 197-223.

[26] Siegel, A. F., Benson, R. H., 1982. A Robust Comparison of Biological Shapes. Biometrics, $38,341-350$.

[27] Day, M. H., Wood, B. A., 1968. Functional affinities of the Olduvai Hominid 8 talus. Man, $3,440-455$.

[28] Gebo, D. L., Schwartz, G. T., 2006. Foot bones from omo: Implications for hominid evolution. American Journal of Physical Anthropology, 129, 499-511.

[29] Wang, C. L., Cheng, C. K., Chen, C. W., Lu, C. M., Hang, Y. S., Liu, T. K., 1995. Contact areas and pressure distributions in the subtalar joint. Journal of Biomechanics, 28, 269-279.

[30] Scheuer, L., Black, S., 2000. Developmental juvenile osteology, London, Academic Press Ltd. 
[31] Humphrey, L. T. 1998. Growth patterns in the modern human skeleton. American Journal of Physical Anthropology, 105, 57-72.

[32] Shea, B. T. 1988. Heterochrony in Primates. In McKinney, L. M. (Eds.) Heterochrony in Evolution. New York, Plenum Press.

\section{Table legends}

Table 1

Population and sex breakdown of astragali specimens included in this study. 


\section{Figure legends}

\section{Figure 1}

Right astragalus morphology. The articular facets of the astragalus are divided up, separately coloured and labelled. The non-articular surfaces are shown in a uniform light yellow. The astragalus is viewed from plantar (A), dorsal (B), lateral (C) and medial (D) aspects.

\section{Figure 2}

Principal Component (PC) 1 scores plotted against centroid size for the PC analysis of unscaled canonical astragali models. The PC analysis was carried out on all 59 specimens together. The plot shows male specimens as filled blue circles and female specimens as filled red squares. The mean scores for males (black M) and females (black F) are shown along with the least squares regression line and corresponding model equation.

\section{Figure 3}

Principal Component (PC) 1 scores plotted against centroid size for the separate PC analyses of un-scaled male and female canonical astragali models. Regression lines, regression equations and mean scores for the separate male and female analyses are shown. Males are shown in blue, females in red.

\section{Figure 4}


The dorsal (A, B) and plantar (C, D) views of the Principal Component (PC) 1 extreme shapes, as identified by the PC analysis of canonical representations of the un-scaled right astragali. The blue model represents the 'small' PC 1 extreme shape. The yellow model represents the 'big' PC 1 extreme shape. In this analysis of human astragali shapes, the big (yellow model) PC 1 extreme model was at the positive end of the PC 1 spectrum, with the small (blue model) PC 1 extreme shape model at the negative end of PC 1.

\section{Figure 5}

Allometric vector maps between Procrustes scaled, translated and rotated Principal Component (PC) 1 extreme shape models (from Figure 4) viewed from dorsal (A) and plantar (B) aspects. Yellow models represent the original 'big' PC 1 extreme, blue models represent the original 'small' PC 1 extreme (as in Figure 4). The red arrows represent the largest 250 allometric vectors and run from small (blue) model to big (yellow) model.

\section{Figure 6}

Allometric vector maps between Procrustes scaled, translated and rotated Principal Component (PC) 1 extreme shape models (from Figure 4) viewed from anterior (A) and posterior (B) aspects. Yellow models represent the original 'big' PC 1 extreme, blue models represent the original 'small' PC 1 extreme (as in Figure 4). The red arrows represent the largest 250 allometric vectors and run from small (blue) model to big (yellow) model.

\section{Figure 7}


Allometric vector maps between Procrustes scaled, translated and rotated Principal Component (PC) 1 extreme shape models (from Figure 4) viewed from medial (A) and lateral (B) aspects. Yellow models represent the original 'big' PC 1 extreme, blue models represent the original 'small' PC 1 extreme (as in Figure 4). The red arrows represent the largest 250 allometric vectors and run from small (blue) model to big (yellow) model.

\section{Figure 8}

Allometric vector maps between Procrustes scaled, translated and rotated Principal Component (PC) 1 extreme shape models for males and females separately. As in Figure 5, the yellow models represent the original 'big' PC 1 extreme, blue models represent the original 'small' PC 1 extreme. The red arrows represent the largest 250 allometric vectors and run from small (blue) model to big (yellow) model.

\section{Video 1}

Dorsal view of the allometric shape change identified by our method in a right astragalus. 3D lines represent the largest 250 allometric vectors. These vectors run from blue dots to yellow dots. Blue points are in the position of the scaled original small PC1 extreme model. Yellow points correspond to the scaled original big PC1 extreme model. Video runs from scaled small to big scaled astragalus shape.

\section{Video 2}

Plantar view of the allometric shape change identified by our method in a right astragalus.

3D lines represent the largest 250 allometric vectors. These vectors run from blue dots to 
yellow dots. Blue points are in the position of the scaled original small PC1 extreme model. Yellow points correspond to the scaled original big PC1 extreme model. Video runs from scaled small to big scaled astragalus shape.

\section{Video 3}

Posterior view of the allometric shape change identified by our method in a right astragalus. $3 \mathrm{D}$ lines represent the largest 250 allometric vectors. These vectors run from blue dots to yellow dots. Blue points are in the position of the scaled original small PC1 extreme model. Yellow points correspond to the scaled original big PC1 extreme model. Video runs from scaled small to big scaled astragalus shape.

\section{Video 4}

Lateral view of the allometric shape change identified by our method in a right astragalus. 3D lines represent the largest 250 allometric vectors. These vectors run from blue dots to yellow dots. Blue points are in the position of the scaled original small PC1 extreme model. Yellow points correspond to the scaled original big PC1 extreme model. Video runs from scaled small to big scaled astragalus shape. 
Table 2

Population and sex breakdown of astragali specimens included in this study.

\begin{tabular}{|l|r|r|r|r|}
\hline & male & female & unknown sex & total \\
\hline Andamanese islander & 4 & 5 & 4 & 13 \\
Australian Aborigine & 3 & 2 & & 5 \\
African Kalahari bushman & & 7 & & 7 \\
Romano British (Poundbury collection) & 7 & 7 & & 14 \\
Industrial British (Spitalfields collection) & 7 & 4 & & 12 \\
Sri Lankan & 3 & 4 & 1 & 8 \\
& & & 1 & \\
total Homo sapiens & 24 & 29 & & \\
\hline
\end{tabular}


Fig 1

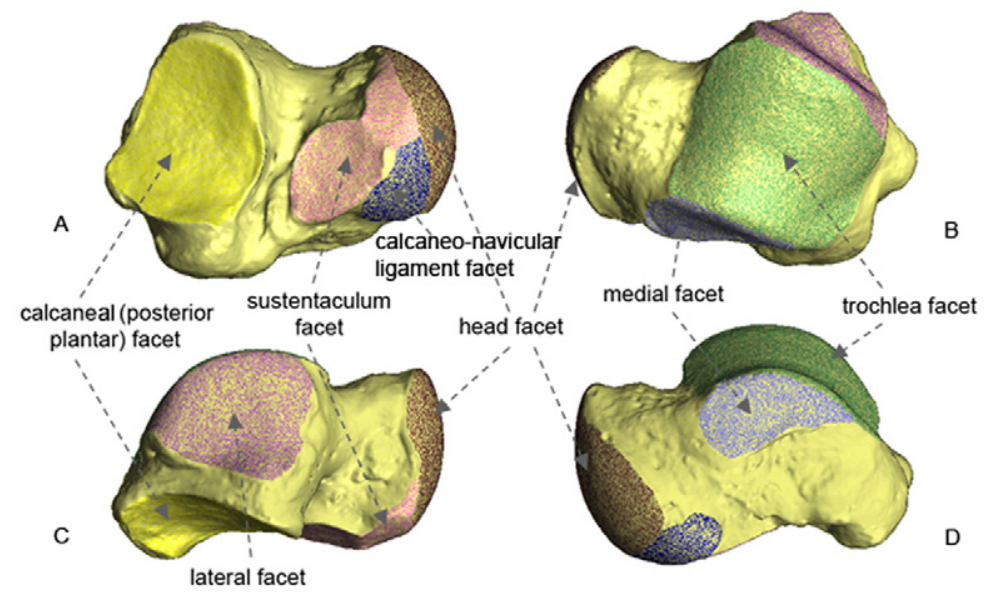


Fig 2

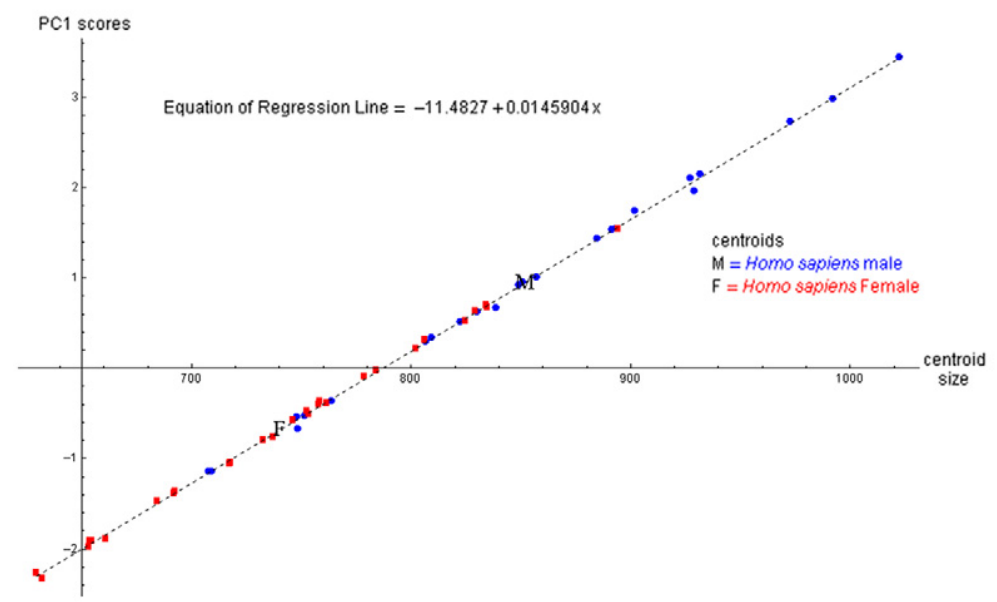


Fig 3

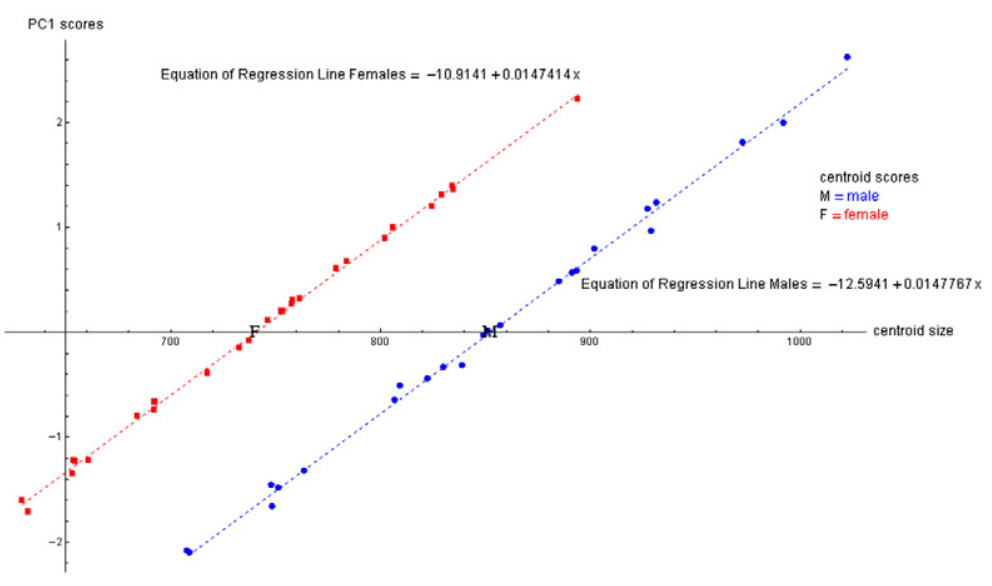


Fig 4

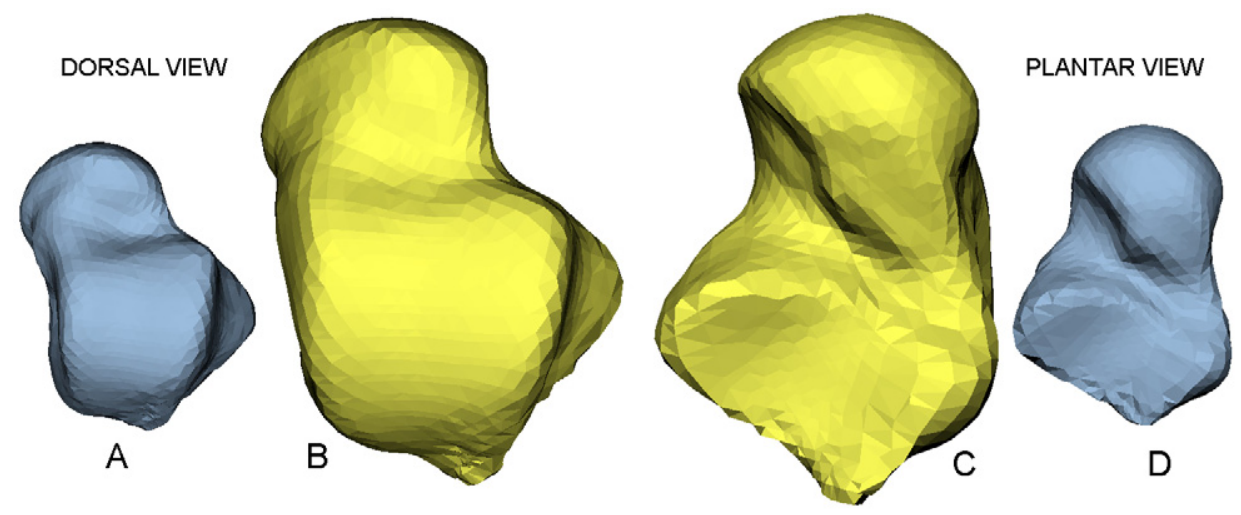


Fig 5
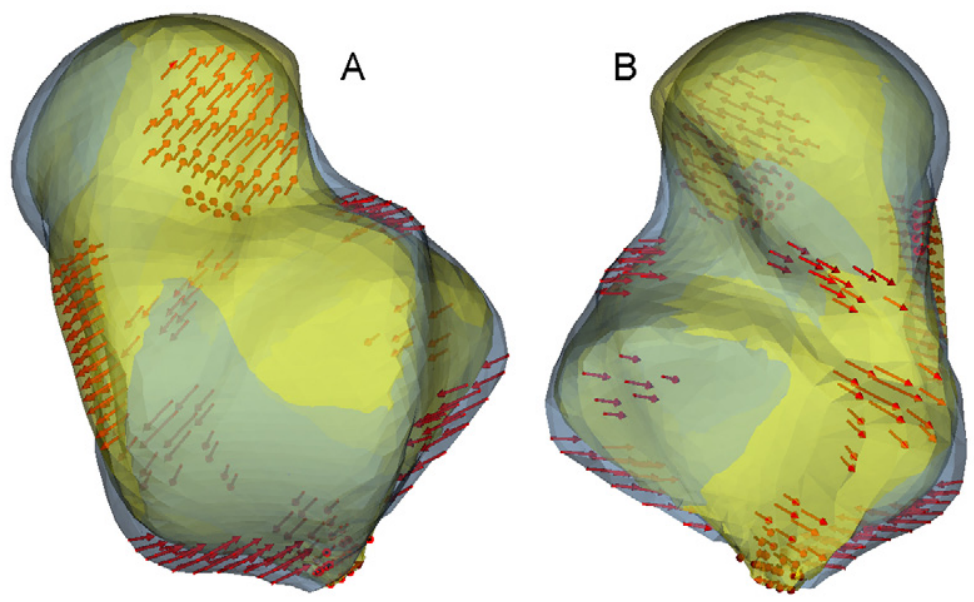
Fig 6
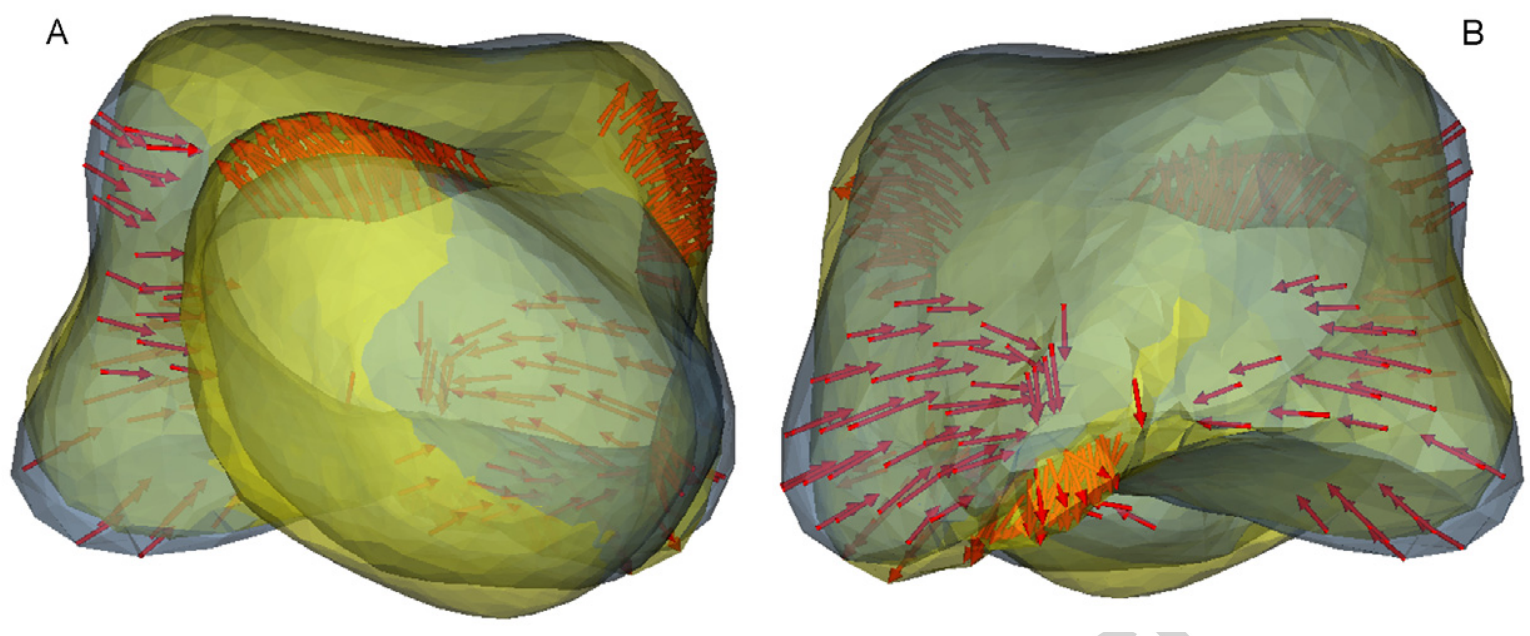

10 
Fig 7
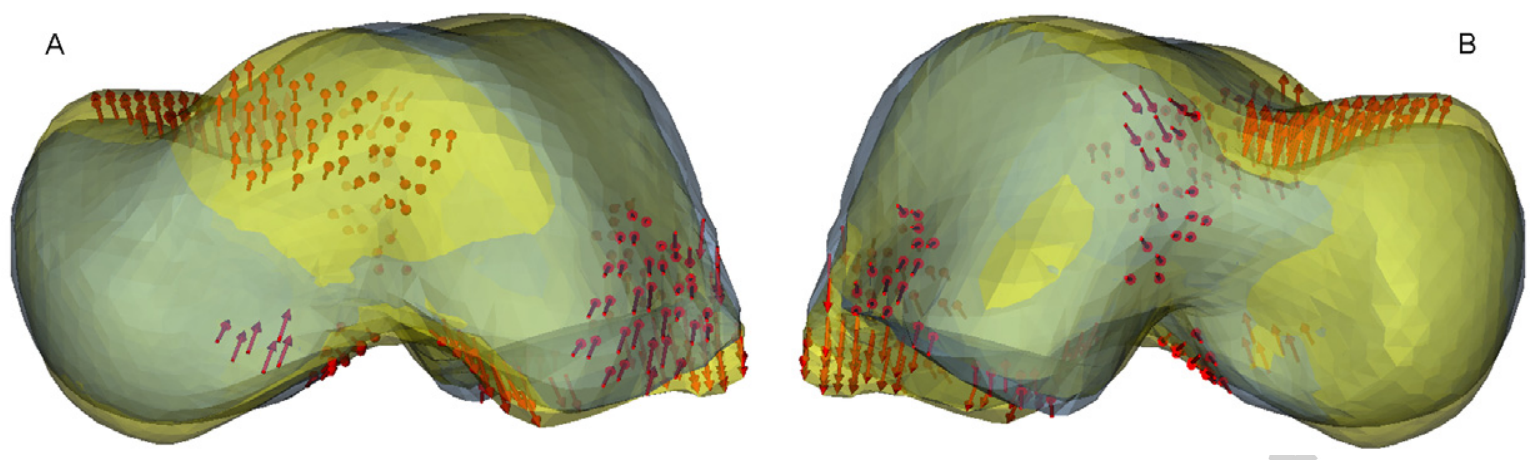
Fig 8

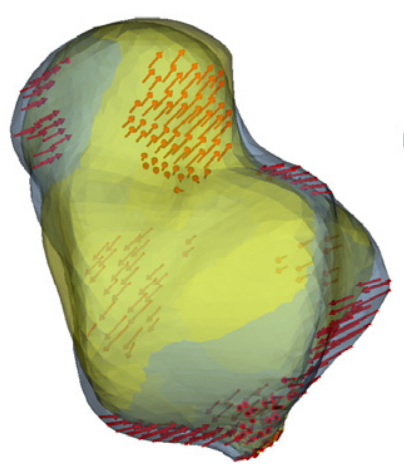

male

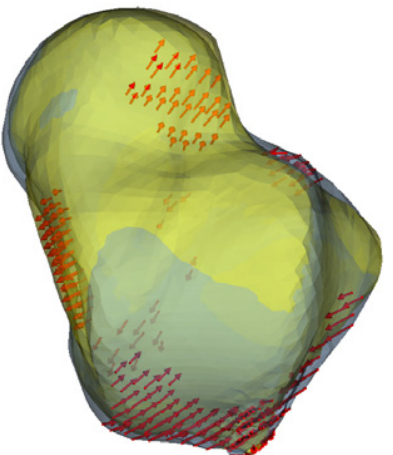

female Dorsal view

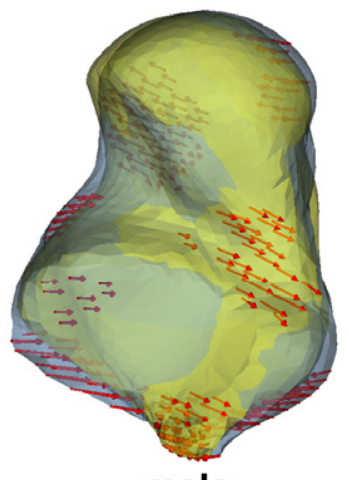

male

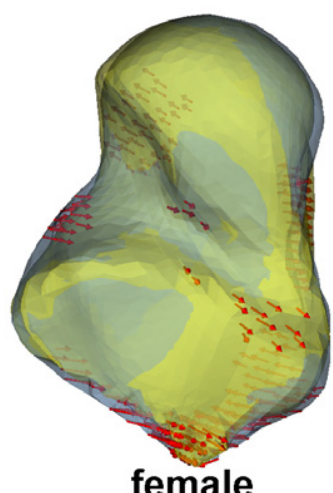

female

Plantar view 